\title{
The Search for Binaries in Post-Asymptotic Giant Branch Stars: Do Binary Companions Shape the Nebulae?
}

\author{
Bruce J. Hrivnak ${ }^{\dagger}$ \\ Department of Physics and Astronomy, Valparaiso University, Valparaiso, IN 46383, USA
}

Binary companions are often invoked to explain the axial and point symmetry seen in the majority of planetary nebulae and proto-planetary nebulae (PPNs). To explore this hypothesis, we have undertaken a long-term (20 year) study of light and velocity variations in PPNs. From the photometric study of 24 PPNs, we find that all vary in brightness, and from a subset of 12 carbon-rich PPNs of F-G spectral type we find periods of 35-155 days, with the cooler having the longer periods. The variations are seen to be due to pulsation; no photometric evidence for binarity is seen. A radial velocity study of a sub-sample of seven of the brightest of these shows that they all vary with the pulsation periods. Only one shows evidence of a longer-term variation that we tentatively identify as being due to a binary companion. We conclude that the present evidence for the binary nature of these PPNs is meager and that any undetected companions of these PPNs must be of low mass $(<0.25 \mathrm{M} \odot$ ) or long period ( $>30$ years).

Keywords: planetary nebulae: general, stars: asymptotic giant branch and post-asymptotic giant branch, stars: binary, stars: variables: other

\section{INTRODUCTION}

\subsection{Motivation for the Study}

The search for binary companions to post-asymptotic giant branch (post-AGB) stars is largely motivated by a desire to better understand the processes that shape the nebulae surrounding these objects. Planetary nebulae (PNs) display nebulae that are classified as round, elliptical, or butterfly based on their appearances. They commonly show an axial or point symmetry. This is not what one would have initially expected for the shape of these nebulae, since they originate in the mass lost by spherical AGB stars. To better understand how this change occurs from spherical mass loss by an AGB star to the axial- and point-symmetric nebula seen in a $\mathrm{PN}$, we are studying proto-PNs (PPNs). These are objects in transition between the AGB and PN phases in the evolution of intermediate- and low-mass stars. They possess detached, expanding gaseous and dusty envelopes that radiate in the mid-infrared (Kwok 1993), and most were initially indentified as a result of the Infrared Astronomical Satellite (IRAS). The central stars are expected to change spectral type from $\mathrm{G}$ to $\mathrm{B}$ supergiants in a timescale of a few thousand years as the stars evolve horizontally at constant luminosity across the Hertzsprung-Russell diagram (Bloecker 1995). Hubble Space Telescope images have resolved these small nebulae and show that PPNs already possess the basic shapes found in PNs (Ueta et al. 2000, Sahai et al. 2007, Siódmiak et al. 2008). Many are bipolar and some show an obscured equatorial region. How are the nebulae shaped?

\subsection{Mechanisms to Shape the Nebulae}

Three general mechanisms have been proposed to shape the nebulae from spherical to axial symmetry. They are as follows:

(c) This is an Open Access article distributed under the terms of the Creative Commons Attribution Non-Commercial License (http://creativecommons.org/licenses/by-nc/3.0/) which permits unrestricted non-commercial use, distribution, and reproduction in any medium, provided the original work is properly cited.

Received Dec 02, 2011 Revised Jan 28, 2012 Accepted Jan 31, 2012 ${ }^{\dagger}$ Corresponding Author

E-mail: bruce.hrivnak@valpo.edu

Tel: +1-219-464-5379 Fax: +1-219-464-5489 
1) Binary companion: a companion could gravitationally focus the mass loss into the orbital plane, forming an equatorial density enhancement, and perhaps a torus, that would then collimate the fast wind producing an axially-symmetric outflow.

2) Rapid rotation: the mass could be preferentially lost in the equatorial plane of the PN during the AGB phase due to a rotationally-induced oblate shape, producing a collimating torus.

3) Magnetic field: the central star could possess a magnetic field that collimates the outflow into bipolar lobes.

While the first of these explicitly requires a binary companion, recent studies indicate that a binary companion is also needed to sustain the shaping mechanism in the other two cases.

Thus the hypothesis of a binary companion is the favored explanation for the shaping of the nebulae (De Marco 2009). In this study, our goal is to observationally investigate this binary hypothesis.

\section{DETECTING BINARIES BY PHOTOMETRIC VARIATIONS}

There are several means by which binaries can be detected photometrically. 1) Eclipses are possible but unlikely unless the orbital period is very short. 2) A hot companion can irradiate the facing hemisphere of the PPN, producing a light variation with a period equal to the orbital period. 3) A companion can tidally-distort the shape of the PPN, producing an ellipsoidal light variation with a period equal to half that of the orbital period. These are the methods used by Bond (2000), Miszalski et al. (2009), and others to identify binary companions to central stars of PNs (CSPNs). This has resulted in the detection of about 40 binary CSPNs; these have periods of about 1-8 days and statistics indicate that about $10-20 \%$ of PNs possess close binaries. 4) Periodic obscuration by circumbinary dust has also been observed in a few binaries of longer period orbits. These included some post-AGB objects like the "Red Rectangle."

To search for these, we have been carrying out a photometric monitoring program of PPNs at the Valparaiso University Observatory, beginning in 1994 and continuing to the present time. The program includes about 24 PPNs with apparent magnitudes ranging from 7 to 14 . The observations are carried out primarily by undergraduate students, using the 0.4- $\mathrm{m}$ telescope and charge-coupled device camera at the campus observatory. This is a unique study; only one other group has been carrying out light curve studies of PPNs, and they have been observing a smaller sample with a smaller temporal baseline (Arkhipova et al. 2000).

In Fig. 1, we show examples of the light curves of three PPNs from 1994 through 2010 (a), and we show on an expanded scale the cyclical variations seen in a typical fouryear interval (b). Period analyses show a dominant period for each of the cooler PPNs of spectral type F-G, with periods ranging from 35 to 155 days. For hotter B types, the light curves vary with still shorter timescales and appear to have multiple periods. The brightnesses vary in a range from 0.15 to $0.6 \mathrm{mag}$. In a study of a sample of 12

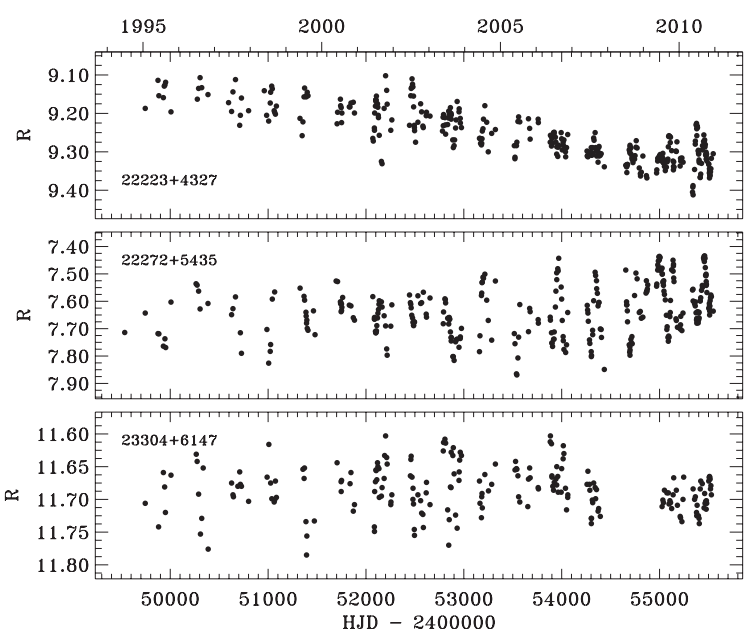

(a)

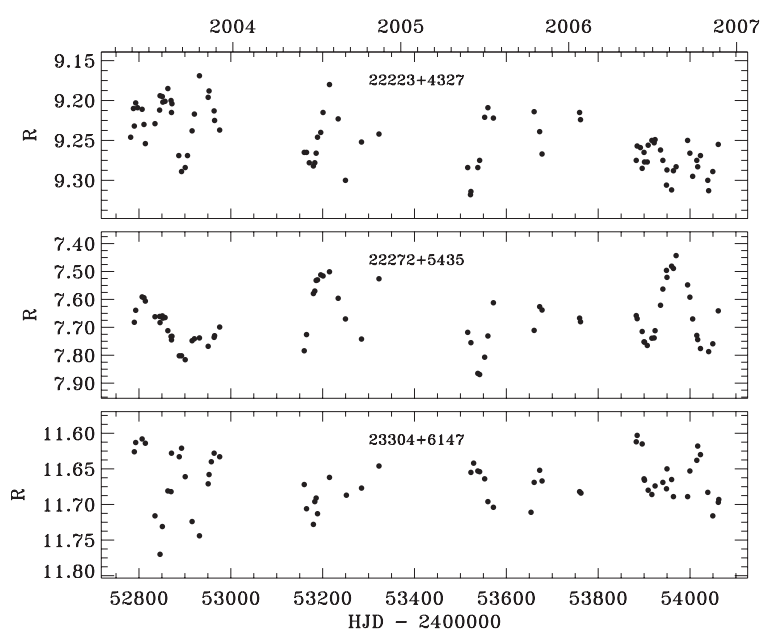

(b)

Fig. 1. (a) Light curves of three representative proto-planetary nebulae from 1993 to 2010, showing the seasonal variations, in some cases superimposed on a general trend in brightness. (b) The light curve variations are shown over a four-year interval, to better display the cyclical nature of the variations. The periods are (top to bottom): IRAS $22223+4327-89 \mathrm{~d} ; 22272+5435-130 \mathrm{~d} ; 23304+4327-85 \mathrm{~d}$. 
carbon-rich PPNs, we find a clear trend of shorter period and smaller amplitude with higher effective temperature (Hrivnak et al. 2010). The light curves show secondary periods often producing a beat pattern and clearly indicate that the light variations are due to pulsation and not binary effects.

One can still consider what constraints are set by this absence of observed binary photometric effects. What would one expect to see if there was a binary companion? We explored this by producing simulated binary light curves using the light curve synthesis program Binary Maker 3 (Bradstreet \& Steelman 2002). If we assume as typical values a mass of the PPN as $0.62 \mathrm{M} \odot$, a companion of $0.5 \mathrm{M} \odot$, and a PPN of $\mathrm{G}$ spectral type with a radius of $90 \mathrm{R} \odot$, and we place the PPN just within its Roche lobe, then the companion orbits with a period of 1 year. At an inclination of $90^{\circ}$, the peak-to-peak light variation would be 0.18 mag in $V$, decreasing with decreasing inclination $\left(0.12 \mathrm{mag}\right.$ at $60^{\circ}$ and $0.04 \mathrm{mag}$ at $\left.30^{\circ}\right)$. In this case, a binary with an inclination of $60^{\circ}$ or greater should be detected photometrically even in the presence of the pulsation. However, the situation is actually not nearly so favorable since in the preceding AGB stage the star had a radius of approximately $400 \mathrm{R} \odot$. To avoid Roche lobe overflow at that stage, a companion would have had a minimum orbital period of 11 years. If we simulate a binary light curve for the present PPN with the companion having an orbital period of 11 years, rather than 1 year, we find a maximum ellipsoidal light variation of only 0.002 mag. There would thus be no expectation to detect this even without the pulsation. So in fact photometric monitoring generally would not be expected to be a fruitful method to detect a binary companion unless there was some orbital migra- tion inward by the companion as the PPN evolved.

\section{DETECTING BINARIES BY RADIAL VELOCITY VARIATIONS}

Radial velocity monitoring holds better promise for detecting binaries with a range of periods from one to several tens of years. Referring to the previous example, a PPN with a mass of $0.62 \mathrm{M} \odot$, a companion of $0.5 \mathrm{M} \odot$, and an orbital period of 1 year would produce a velocity variation of $13 \mathrm{~km} \mathrm{~s}^{-1}$ if viewed in the orbital plane. This would be reduced by the sine of the inclination for other viewing angles, but this only amounts to a factor of 0.5 for an inclination of $30^{\circ}$. Even with an orbital period of 11 years, the velocity amplitude is $6.3 \mathrm{~km} \mathrm{~s}^{-1}$ and with a period of 30 years, the amplitude is $4.5 \mathrm{~km} \mathrm{~s}^{-1}$, values that one can hope to detect. However, detectability also depends on the amplitude of the intrinsic pulsation.

We have been carrying out a program to search for binary velocity variations among a sample of the seven brightest PPNs with F-G spectral type. These have many sharp metallic lines and constitute a much more favorable sample to search for velocity variations than do the hot CSPNs with their fewer and broader lines. Our observations are being carried out with the Coudé spectrograph at the 1.2-m telescope at the Dominion Astrophysical Observatory (DAO), Victoria, Canada. An initial study was carried out from 1991 to 1995, in which we detected pulsational variations of $\sim 10 \mathrm{~km} \mathrm{~s}^{-1}$ peak to peak, with periods similar to those derived from the light curve monitoring. An example is shown in Fig. 2.

Observations were re-initiated in 2007 and are con-

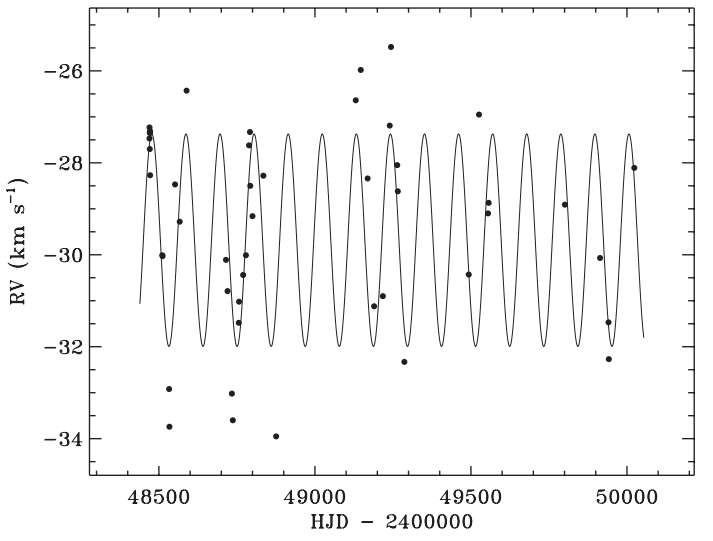

(a)

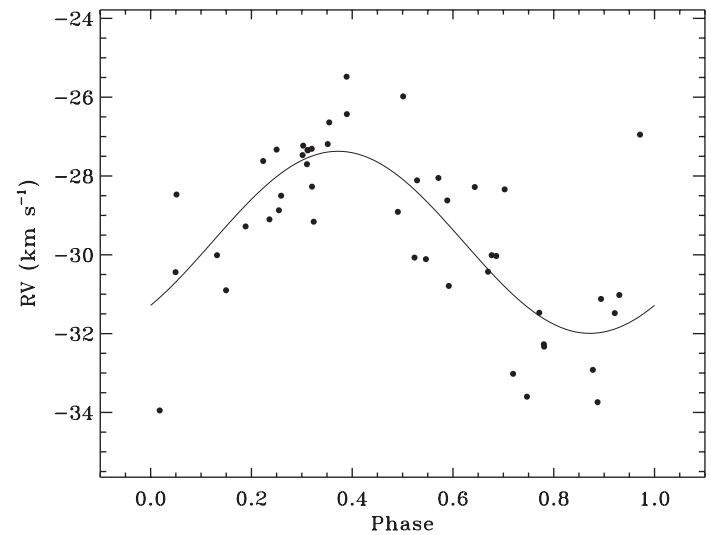

(b)

Fig. 2. (a) Radial velocity curve of IRAS $18095+2704$ from our 1991-1995 observations, showing a sine-curve fit to the periodic pulsational variations. (b) Radial velocity curve phased to the pulsation period of 110 days. 
tinuing in our search to detect binaries. We presently have 40-100 observations of each of these PPNs, with a precision of $\sim 0.6 \mathrm{~km} \mathrm{~s}^{-1}$. However, only in one case, IRAS $22272+5435$, has evidence been found for velocity change beyond pulsation. This is shown in Fig. 3 where we have also included some available observations by others (primarily Zăcs et al. 2009). A systematic difference of $2.6 \mathrm{~km}$ $\mathrm{s}^{-1}$ is seen between the early (1989-1995) and later (20052010) observations. If we assume that these variations are due to a long-period binary companion, they constrain the period to be longer than the 22 year interval of the observations. Assuming a PPN mass of $0.62 \mathrm{M} \odot$, an amplitude of half of the velocity difference $\left(1.3 \mathrm{~km} \mathrm{~s}^{-1}\right)$, a period of 22 years, and a circular orbit, we can solve for the mass of the secondary as a function of orbital inclination. From a model based on mid-infrared imaging of IRAS $22272+5435$, an inclination of $25^{\circ}$ has been determined (Ueta et al. 2001). This leads to a secondary of mass 0.27 $\mathrm{M} \odot$; a longer period would result in an even lower mass. A circular orbit leads to a separation of $8 \mathrm{AU}$ or $1700 \mathrm{R} \odot$, putting the secondary well outside of the Roche lobe even at the tip of the AGB.

The other six show no long-term variations beyond the pulsations. Might there be some bias in the choice of these targets? All seven of the PPNs possess axial symmetric nebulae surrounding the bright central star. Indeed, in choosing the brightest ones (for practical reasons), they are more likely to be seen at low orbital inclinations (more pole-on), when the torus is not obscuring the star. Nevertheless, one can still set significant constraints on the properties of any undetected companions. We have explored this for various values of $\mathrm{M}_{2}$ and $\mathrm{P}$ over a range of inclinations. We find that any undetected binary companions must have low masses or long periods or both (Hrivnak et al. 2011).

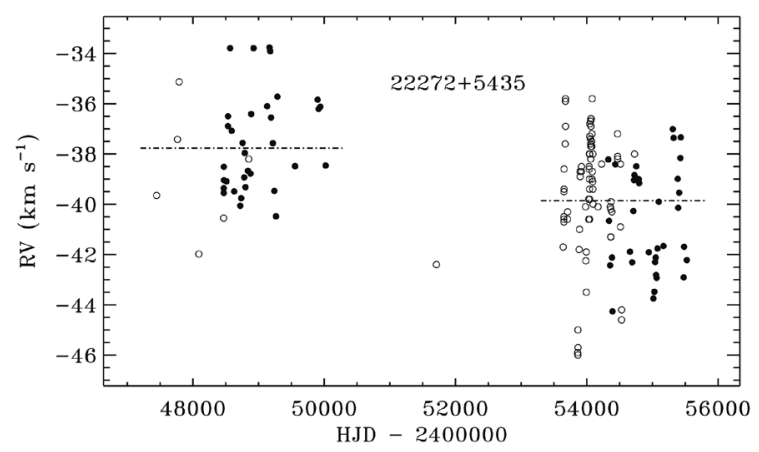

Fig. 3. The radial velocity curve of IRAS $22272+545$. Filled circles represent our observations and open circles those of others. The two dashed lines represent the average values in the two intervals of 19891995 and 2005-2010; these differ by $2.6 \mathrm{~km} \mathrm{sec}^{-1}$.

\section{RESULTS AND CONCLUSIONS}

The results of this study are that all of the 24 PPNs observed show photometric variations due to pulsation but that none of them show photometric evidence of being binaries. On the basis of the radial velocity monitoring, only one of the seven is tentatively identified as being a spectroscopic binary; it has an orbital period greater than 22 years. The absence of evidence for binarity in the other six sets constraints on any binary companions: they must have low mass $(<0.25 \mathrm{M} \odot)$ or long periods ( $>30$ years). They could be brown dwarfs or even super-Jupiters. The CSPNs that others have detected photometrically have periods on the order of days and are thought to have evolved through a common envelope phase. One might wonder if our PPNs happen to be in such a phase that has rendered the companions unobservable. However, it is extremely unlikely that six of seven would be in the common envelope phase, which is expected to last only a short time, on the order of a year. This study comprises the first systematic search for binaries among PPNs, and it seems fair to say that the results do not support the binary hypothesis. While they do not rule out the presence of binary companions, any binary present must have low mass $(<0.25 \mathrm{M} \odot$ ) or long periods ( $>30$ years). They could be brown dwarfs or even super-Jupiters. Should such extreme companions be present, their efficacy as shaping mechanisms will by greatly reduced.

This leaves us with the question of what does shape the nebulae in PNs and PPNs. The seven objects that we have monitored for both photometric and for radial velocity variations give strong evidence of being PPNs, and they all show axial symmetry, implying that the shaping has started. However, it appears that they do not pass through the common envelope phase since it is so short. This suggests that there may be two ways to form PPNs and shape the nebulae: 1) by common envelope evolution, which leads to the ejection as well as the shaping of the envelope, resulting in a short-period binary CSPN; 2) by non-common envelope evolution, which might involve a distant or low-mass companion or might result from a single, pulsating AGB star.

We are continuing to monitor these and additional PPNs to further investigate and constrain binary parameters. We are also studying their pulsation, which is very interesting in its own right and has the potential to give us information on the interior structure of these evolving stars. 


\section{ACKNOWLEDGMENTS}

I want to begin by acknowledging my debt to R. H. Koch, my thesis advisor, collaborator, and friend. Bob provided a model of one who worked carefully and diligently, who respected the data, and who sought to place the results in the larger astrophysical context. I thank my collaborators at the DAO, D. Bohlender, A. Woodsworth, S. Morris, and C. Scarfe (University of Victoria), and I acknowledge the efforts of many Valparaiso University undergraduate summer research assistants who have photometrically monitored these PPNs. The assistance of W. Lu in much of this research is gratefully acknowledged. This research has been made possible by the long-term funding of the National Science Foundation (AST 9018032, 9315107, 9900846, 0407087, 1009974) and the Indiana Space Grant Consortium.

\section{REFERENCES}

Arkhipova VP, Ikonnikova NP, Noskova RI, Sokol GV, UBV observations of post-AGB supergiants and peculiarities of their photometric variability, AstL, 26, 609-617 (2000). http://dx.doi.org/10.1134/1.1307895

Bloecker T, Stellar evolution of low- and intermediate-mass stars. II. Post-AGB evolution, A\&A, 299, 755-769 (1995).

Bond HE, Binarity of central stars of planetary nebulae, in Asymmetrical planetary nebulae II: from origins to microstructures, ASP Conference Series, Vol. 199, eds. Kastner JH, Soker N, Rappaport S (Astronomical Society of the Pacific, San Francisco, CA, 2000), 115-124.

Bradstreet DH, Steelman DP, Binary Maker 3.0: an interactive graphics-based light curve synthesis program written in Java, Bull AAS, 34, 1224 (2002).

De Marco $\mathrm{O}$, The origin and shaping of planetary nebulae: putting the binary hypothesis to the test, PASP, 121, 316-
342 (2009). http://dx.doi.org/10.1086/597765

Hrivnak BJ, Lu W, Bohlender D, Morris SC, Woodsworth AW, et al., Are proto-planetary nebulae shaped by a binary? Results of a long-term radial velocity study, ApJ, 734, 25 (2011). http://dx.doi.org/10.1088/0004-637X/734/1/25

Hrivnak BJ, Lu W, Maupin RE, Spitzbart BD, Variability in proto-planetary nebulae. I. Light curve studies of 12 carbon-rich objects, ApJ, 709, 1042-1066 (2010). http:// dx.doi.org/10.1088/0004-637X/709/2/1042

Kwok S, Proto-planetary nebulae, ARA\&A, 31, 63-92 (1993). http://dx.doi.org/10.1146/annurev.aa.31.090193.000431

Miszalski B, Acker A, Moffett AFJ, Parker QA, Udalski A, Binary planetary nebulae nuclei towards the Galactic bulge. I. Sample discovery, period distribution, and binary fraction, A\&A, 496, 813-825 (2009). http://dx.doi.org/ $10.1051 / 0004-6361 / 200811380$

Sahai R, Morris M, Sánchez Contreras C, Claussen M, Preplanetary nebulae: a Hubble Space Telescope imaging survey and a new morphological classification system, AJ, 134, 2200-2225 (2007). http://dx.doi.org/10.1086/522944

Siódmiak N, Meixner M, Ueta T, Sugerman BEK, Van de Steene GC, et al., Hubble Space Telescope snapshot survey of post-AGB objects, ApJ, 677, 382-400 (2008). http://dx.doi.org/10.1086/529115

Ueta T, Meixner M, Bobrowsky M, A Hubble Space Telescope snapshot survey of proto-planetary nebula candidates: two types of axisymmetric reflection nebulosities, ApJ, 528, 861-884 (2000). http://dx.doi.org/10.1086/308208

Ueta T, Meixner M, Hinz P, Hoffmann WF, Brandner W, et al., Subarcsecond mid-infrared structure of the Dust Shell around IRAS 22272+5435, ApJ, 557, 831-843 (2001). http://dx.doi.org/10.1086/322259

Zăcs L, Sperauskas J, Musaev FA, Smirnova O, Yang TC, et al., Dynamical phenomena in the atmosphere of the protoplanetary nebula IRAS 22272+5435, ApJ, 695, L203-L207 (2009). http://dx.doi.org/10.1088/0004-637X/695/2/L203 